\title{
Measurements of Stellar Properties through Asteroseismology: A Tool for Planet Transit Studies
}

\author{
Hans Kjeldsen ${ }^{1}$, Timothy R. Bedding ${ }^{2}$ and \\ Jørgen Christensen-Dalsgaard ${ }^{1}$ \\ ${ }^{1}$ Danish AsteroSeismology Centre, Department of Physics and Astronomy, University of \\ Aarhus, Ny Munkegade, DK-8000 Aarhus C, Denmark \\ email: hans@phys.au.dk and jcd@phys.au.dk \\ ${ }^{2}$ Institute of Astronomy, School of Physics A28, University of Sydney, NSW 2006, Australia \\ email: bedding@physics.usyd.edu.au
}

\begin{abstract}
Oscillations occur in stars of most masses and essentially all stages of evolution. Asteroseismology is the study of the frequencies and other properties of stellar oscillations, from which we can extract fundamental parameters such as density, mass, radius, age and rotation period. We present an overview of asteroseismic analysis methods, focusing on how this technique may be used as a tool to measure stellar properties relevant to planet transit studies. We also discuss details of the Kepler Asteroseismic Investigation - the use of asteroseismology on the Kepler mission in order to measure basic stellar parameters. We estimate that applying asteroseismology to stars observed by Kepler will allow the determination of stellar mean densities to an accuracy of $1 \%$, radii to $2-3 \%$, masses to $5 \%$, and ages to $5-10 \%$ of the main-sequence lifetime. For rotating stars, the angle of inclination can also be determined.
\end{abstract}

\section{Introduction}

Asteroseismology - the study of stellar oscillations - is a relatively new and growing research field in astrophysics. The analysis of frequencies and other properties of stellar oscillations allows us to constrain fundamental parameters of stars such as density, mass, radius, age, rotation period and chemical composition.

Oscillations are found in stars of most masses and essentially all stages of evolution. The amplitudes and phases are controlled by the energetics and dynamics of the nearsurface layers. The frequencies are determined by the internal sound-speed and density structure of the star, as well as rotation and (in some cases) magnetic fields. Observationally, the frequencies can be determined with exceedingly high accuracy compared to any other quantity relevant to the internal properties of the stars. Analysis of the observed frequencies, including comparison with computed stellar models, allows determination of the properties of the stellar interiors and tests of the physics used in the model computation, applied under extreme conditions that cannot be matched in terrestrial laboratories.

Rotation induces fine structure in the frequency spectrum, in the form of rotational splitting. The observed frequencies are determined by averages over the stellar interior of the rotation rate, which in general varies with position within the star. By comparing with independent determinations of the surface rotation rate, or from rotational splittings for a sufficient broad variety of modes, information about this variation can be obtained. 
Some of the basic parameters that may be obtained from asteroseismology are crucial for understanding the fundamental properties of exoplanet systems. Hence, asteroseismology will potentially be an excellent tool for characterizing planet transit systems and, specifically, it will be able to measure stellar radii to a relative accuracy of $2-3 \%$.

For a recent review on the present state of asteroseismology, see Aerts et al. (2008) and Bedding \& Kjeldsen (2008). In Figure 1 we show examples of power spectra for four different stars. In each case, we can clearly see the excess energy arising from stellar oscillations. The detailed properties of those oscillations form the observational basis for the asteroseismic analysis.

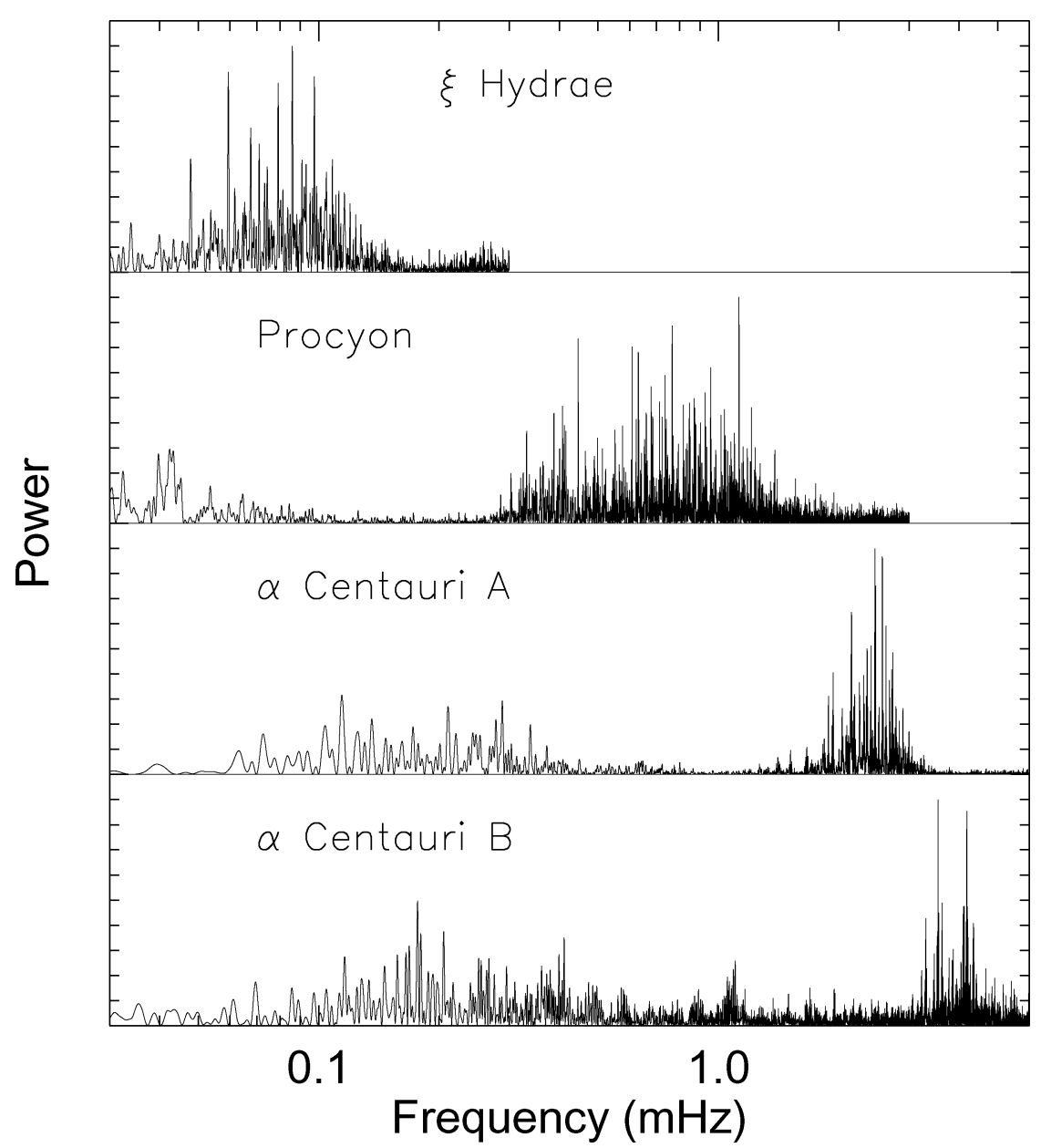

Figure 1. Power spectra of time series observed in radial velocity for the four stars $\xi \mathrm{Hy}$ drae (G7 III; Frandsen et al. 2002), Procyon (F5 IV; Arentoft et al. 2008), $\alpha$ Centauri A (G2 V; Bedding et al. 2004) and $\alpha$ Centauri B (K2 V; Kjeldsen et al. 2005). The oscillation periods for the four stars are: around 3-4 hours for $\xi$ Hydrae, 20-25 minutes for Procyon, 7 minutes for $\alpha$ Cen A and 4 minutes for $\alpha$ Cen B. These differences, and those in the detailed structure of the power spectra, reflect the differences in stellar properties (radius, mass, surface temperature and age). Note that the vertical scales are normalized - the actual amplitudes decrease from top to bottom in the figure. 


\section{The relation between the stellar properties and the frequencies}

To obtain information about stellar properties and the underlying physics, the observed oscillation frequencies must be compared with those computed from models. Major uncertainties affect the treatment of hydrodynamical processes in stellar interiors. These include convective motions, with possible overshoot into the surrounding convectively stable regions, and flows and instabilities related to internal rotation. Furthermore, the evolution of rotation as a star evolves, with internal redistribution and possibly surface loss of angular momentum, has potentially substantial - and highly uncertain - effects on the evolution of the star. Despite those uncertainties in the knowledge of some of the detailed physics, we are able to obtain quite detailed basic properties for a number of general stellar parameters such as density and the amount of hydrogen in the core. The frequencies of stellar p-mode oscillations are related to the sound travel time across the star. Since the sound speed, $c$, is given by

$$
c^{2}=\frac{\Gamma_{1} P}{\rho} \simeq \frac{5}{3} \frac{k_{B} T}{\mu m_{u}},
$$

(where the approximation assumes an ideal gas), we are basically measuring the average ratio between pressure and density in the stellar interior.

The exact frequencies of stellar oscillations depend on the detailed structure of the star and on the physical properties of the gas. However, one may use asymptotic theory to

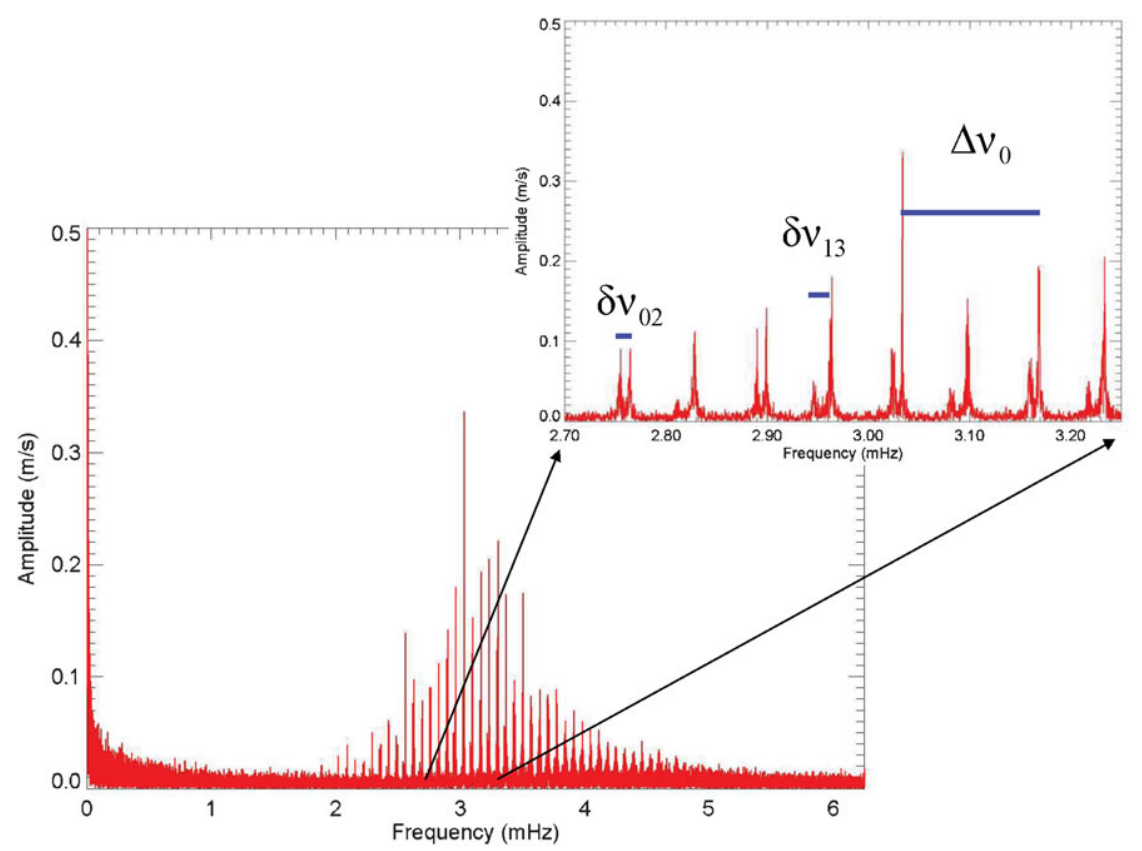

Figure 2. The power spectrum of oscillations in the Sun. The data are full-disk radial velocity measurements obtained over 30 days using the GOLF instrument on board the SoHO spacecraft (Ulrich et al. 2000, García et al. 2005). The inset shows the details of the p-mode structure, and we indicate the so-called large and small separations, which contain information on the basic stellar properties. 
derive a simple relation for the mode frequencies (see, for example, Christensen-Dalsgaard 2004). Each oscillation mode is characterized by three integers: the radial order $n$, the angular degree $l$ and the azimuthal order $m$. The results of the asymptotic analysis for a non-rotating star give the mode frequencies as

$$
\nu_{n, l}=\Delta \nu\left(n+\frac{1}{2} l+\epsilon\right)-l(l+1) D_{0} .
$$

Here, $\Delta \nu$ (the so-called large separation) depends on the average stellar density, $D_{0}$ is sensitive to the sound speed near the core and $\epsilon$ is sensitive to the surface layers. It is conventional to define $\delta \nu_{02}$, the so-called small separation, as the frequency spacing between adjacent modes with $l=0$ and $l=2$. These separations are indicated for the Sun in Figure 2, together with the similar quantity $\delta \nu_{13}$. We can further define $\delta \nu_{01}$ to be the amount by which $l=1$ modes are offset from the midpoint between the $l=0$ modes on either side. If the asymptotic relation holds exactly, then it follows that $D_{0}=\frac{1}{6} \delta \nu_{02}=\frac{1}{2} \delta \nu_{01}=\frac{1}{10} \delta \nu_{13}$.

In practice, the asymptotic relation does not hold exactly, even for the Sun. For example, the large separation depends on $l$. However, we can define average values for $\Delta \nu$

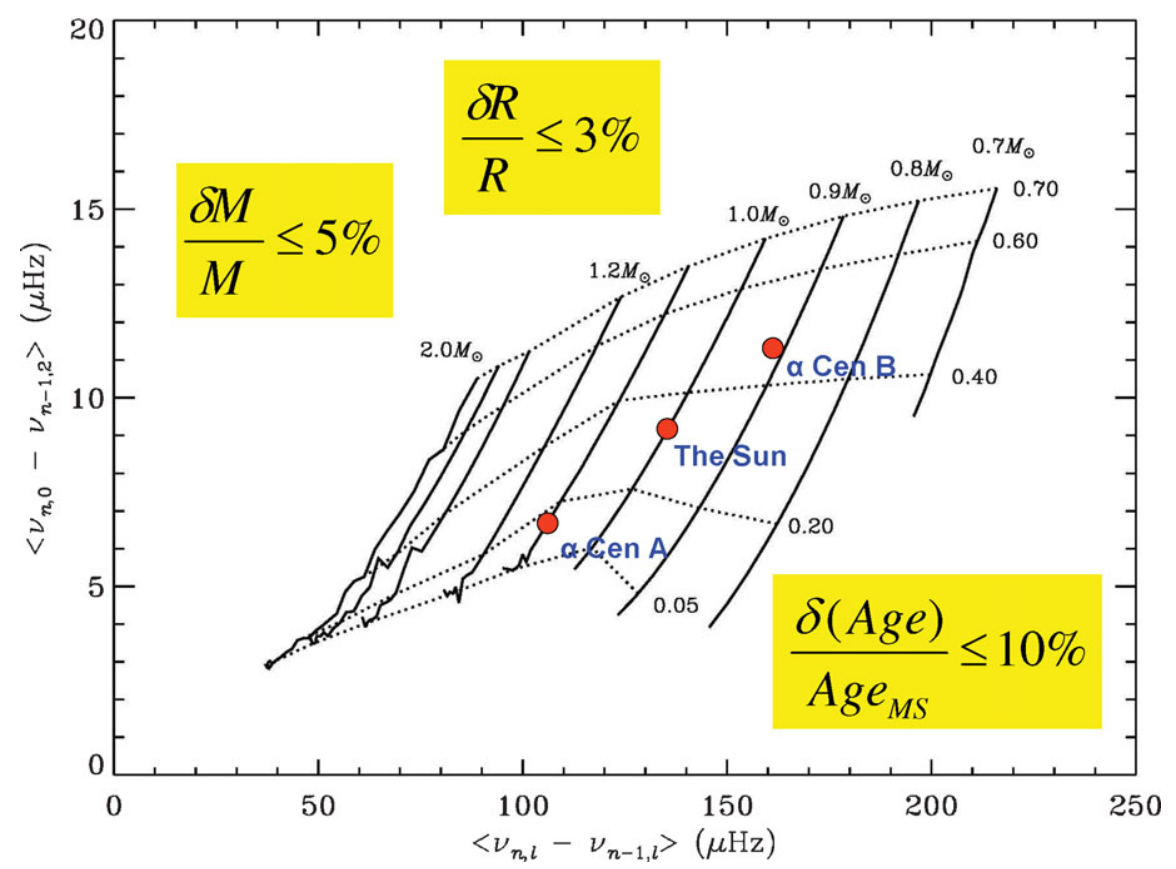

Figure 3. The so-called asteroseismic HR-diagram (Christensen-Dalsgaard 1993), where the axes are the large and small frequency separations $\left(\Delta \nu\right.$ and $\left.\delta \nu_{02}\right)$. The solid lines are evolutionary tracks (for fixed mass) and the dashed lines show constant core-hydrogen content, which is related to the age. Based on simulations we have estimated the uncertainties for the measurements of mass, radius and stellar age using the seismic HR-diagram to be $5 \%$ for the mass, $2-3 \%$ for the radius and $5-10 \%$ for the age (relative to the total time on the main sequence). The mean densities can be measured to $1 \%$. The positions of the Sun and $\alpha$ Centauri A and B are indicated, and it can be seen that $\alpha$ Cen A is more evolved than the Sun while $\alpha$ Cen B is less evolved, in agreement with traditional modeling. 
and $\delta \nu_{02}$, and then can calculate how those parameters depend on the stellar properties and the stellar evolutionary stage. An example of such a calculation is shown by the lines in Figure 3. In this figure we also show the measured position of the Sun, together with that of $\alpha$ Centauri A (Bedding et al. 2004) and $\alpha$ Centauri B (Kjeldsen et al. 2005). This diagram confirms that $\alpha$ Cen A has a higher mass than the Sun and is more evolved, while $\alpha$ Cen B has a lower mass than the Sun and is less evolved.

The exact positions of the tracks shown in Figure 3 will depend on the detailed physical properties, including chemical composition. However, the basic stellar parameters can still be estimated with some robustness. We have used simulations to estimate the accuracy with which various parameters can be measured, assuming that the stellar temperature is already known to within $150-200 \mathrm{~K}$ and the heavy-element abundance is known to within a factor of two. We find that

- stellar mean densities can be measured to $1 \%$,

- stellar radii can be measured to $2-3 \%$,

- stellar masses can be measured to $5 \%$ and

- stellar ages can be measured to $5-10 \%$ of the main-sequence lifetime.

\section{Stellar Rotation}

Equation 2.2 applies to a non-rotating star, for which the frequency of each mode depends only on the radial order $n$ and the angular degree $l$. For a rotating star, the

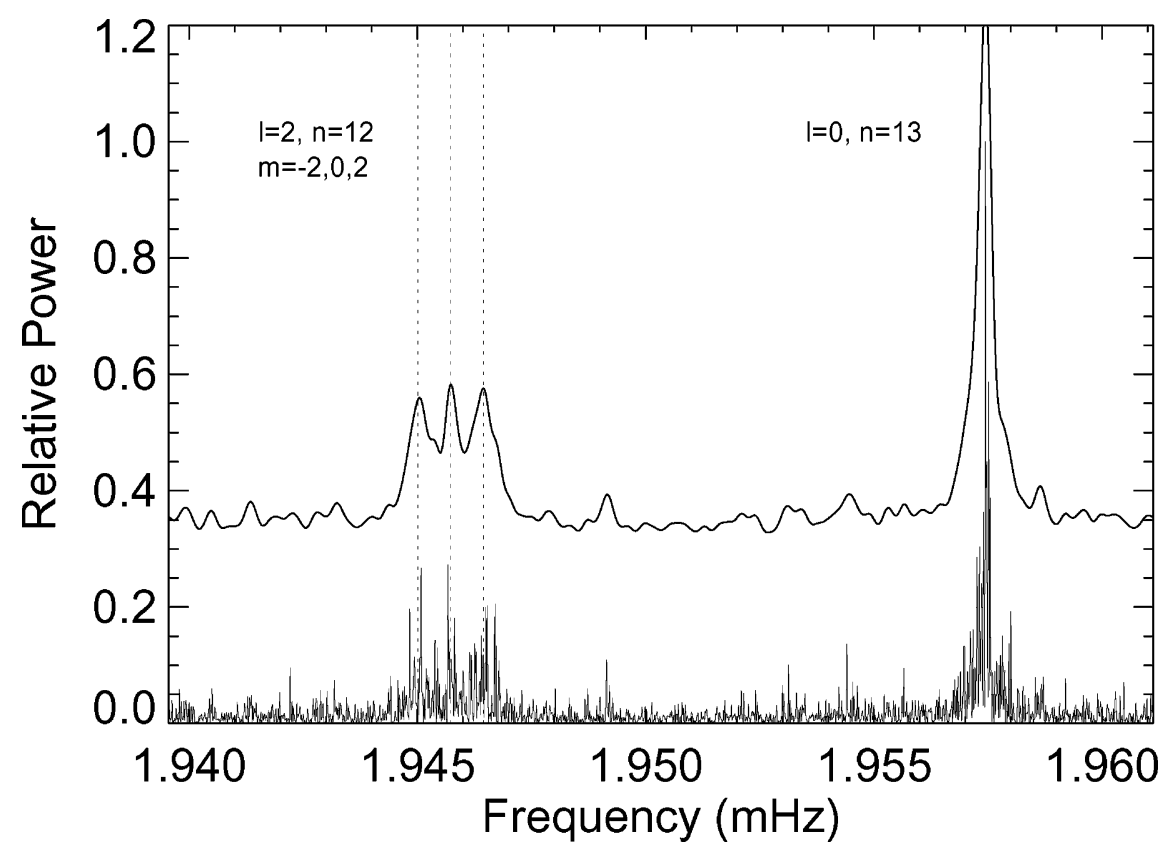

Figure 4. A close-up of the power spectrum of solar oscillations, from full-disk radial velocity measurements. The data are based on 805 days of observing the Sun using the GOLF instrument on board the SoHO spacecraft (Ulrich et al. 2000, García et al. 2005). We show a small region of the power spectrum just below $2 \mathrm{mHz}$. In this region one can see the power from modes with $l=2, n=12$ and $l=0, n=13$. The $l=2$ multiplet has 5 components $(m=0, \pm 1, \pm 2)$ but, due to the inclination of the rotation axis of the Sun, only modes with $m=-2,0$ and 2 are visible. The strength of the individual $m$-components can be used to measure the inclination axis of the stellar rotation. Modes with $l=0$ have only $m=0$ and are therefore not split by rotation. The upper curve is a smoothed version of the power spectrum, shifted vertically for clarity. 
frequencies also depend on the azimuthal degree, $m$ (which takes on values of $m=$ $0, \pm 1, \ldots, \pm l)$. Provided the rotation is slow, the frequencies are well approximated by

$$
\nu_{n, l}=\Delta \nu\left(n+\frac{1}{2} l+\epsilon\right)-l(l+1) D_{0}+m \Delta \nu_{\mathrm{ROT}} .
$$

Here, $\Delta \nu_{\mathrm{ROT}}$ is related to the inverse of the average of the internal rotation period and we should therefore be able to infer stellar rotation periods directly from the oscillation frequencies.

In principle, the inclination of the rotation axis can also be determined because it affects the relative amplitudes of the different azimuthal degrees. This effect is discussed in detail by Gizon \& Solanki (2003). For example, they show that for intensity observations (oscillations detected in photometry) the dipole multiplets $(l=1, m=-1,0,1)$ will have relative mode powers given by

$$
\begin{gathered}
P_{l=1, m=0}=\cos ^{2} i, \\
P_{l=1, m= \pm 1}=\frac{1}{2} \sin ^{2} i .
\end{gathered}
$$

In Figures 4, 5 and 6 we show examples of rotational splitting for the Sun. The splitting of the $l=1$ and 2 modes is clearly seen. The relative strengths of the different $m$ components is a result of the observed inclination of the solar rotation axis. Since we observe the Sun in the equatorial plane, we have almost no sensitivity to the modes with $l=1, m=0$ and those with $l=2, m= \pm 1$. The structure of the power spectrum therefore indicates that the solar interior is rotating in the surface equatorial plane of the Sun, approximately corresponding to the plane of the ecliptic.

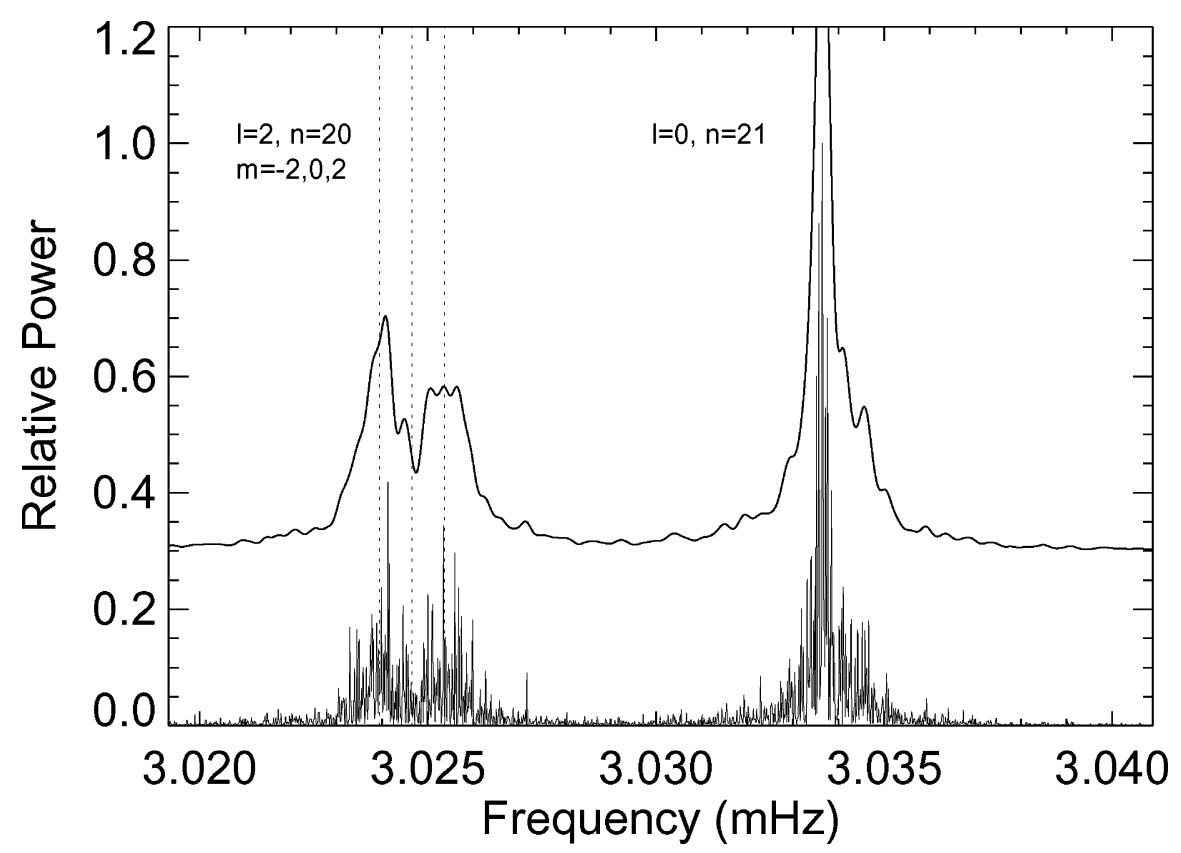

Figure 5. Same as Figure 4, but around a frequency of $3 \mathrm{mHz}$. We see the multiplet with $n=20$ and $l=2$, and the singlet with $n=21$ and $l=0$. 


\section{The Kepler Asteroseismic Investigation}

The NASA Kepler mission (Borucki et al. 2008) will fly a wide-field Schmidt camera with $0.95 \mathrm{~m}$ aperture, staring at a single field continuously for at least 3.5 years. Although the mission's principal aim is to locate transiting extrasolar planets, it will provide an unprecedented opportunity to make asteroseismic observations of a wide variety of stars. This will give the opportunity to measure global properties and internal structure for a large number of stars across a broad range of different types. Plans are now being developed to exploit this opportunity to the fullest. In particular, asteroseismic analysis of Kepler data will provide an accurate determination of the radius for a large fraction of the stars found to host planetary systems, as determined from the transit analysis of the Kepler data, as well as estimates of the ages of the systems. Through investigation of a broad range of stars, the asteroseismic investigation will also substantially improve our understanding of general stellar evolution, and hence strengthen the use of such modelling to further constrain the properties and evolution of the stars and systems investigated in the Kepler extra-solar planet programme. The purpose of the Kepler Asteroseismic Investigation (e.g., Christensen-Dalsgaard et al. 2007) is to ensure that full use is made of this potential to benefit the Kepler investigations of extra-solar planetary systems.

Kepler will give the possibility of observing at two cadences (1 minute and 30 minutes). Due to the high frequency of the stellar oscillations Kepler will not be able to make asteroseismic observations of all the 170,000 stars observed in long cadence mode (30 minute cadence). We will therefore need a target selection programme in order to optimize the asteroseismic part of the Kepler programme. Based on simulations, we have estimated that seismology can be done on unevolved main-sequence stars down to magnitude

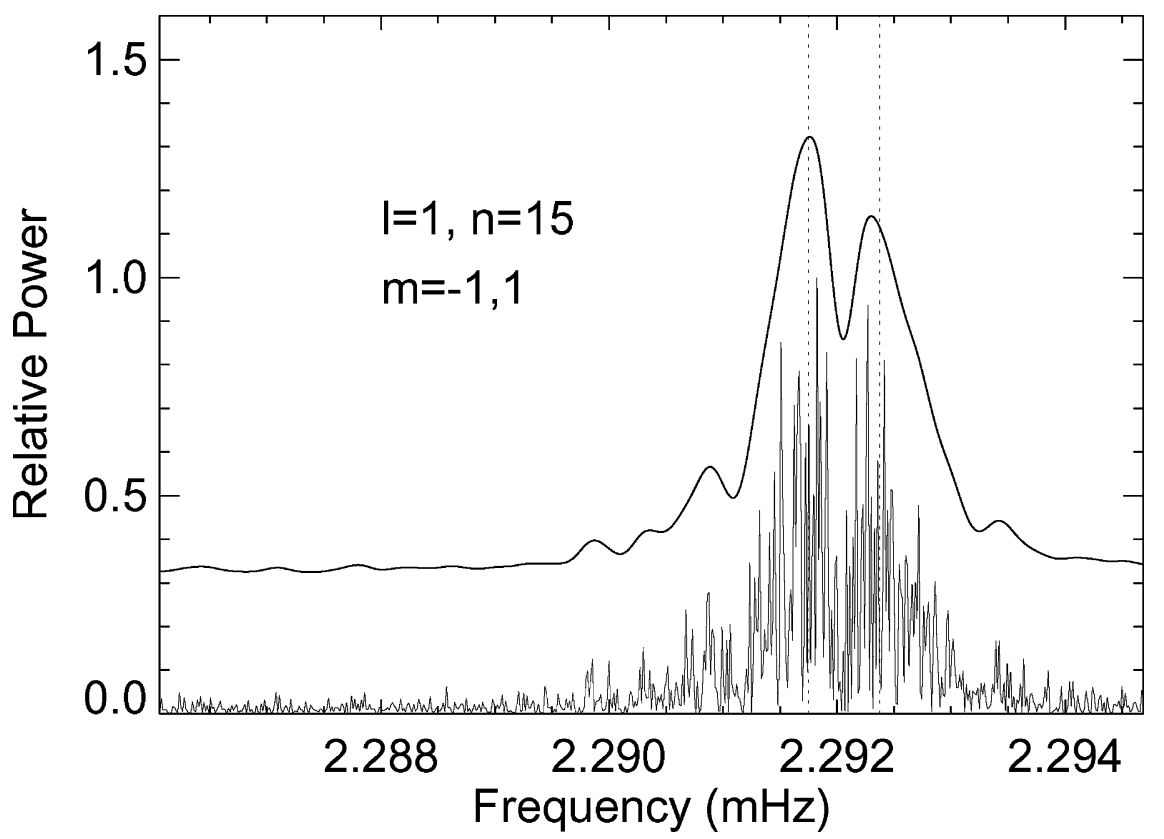

Figure 6. Same as Figure 4, but at a frequency just below $2.3 \mathrm{mHz}$. In this region one can see the power from the triplet with $l=1, n=15, m=0, \pm 1$. Due to the inclination of the rotation axis of the Sun, only modes with $m=-1$ and 1 are visible. 


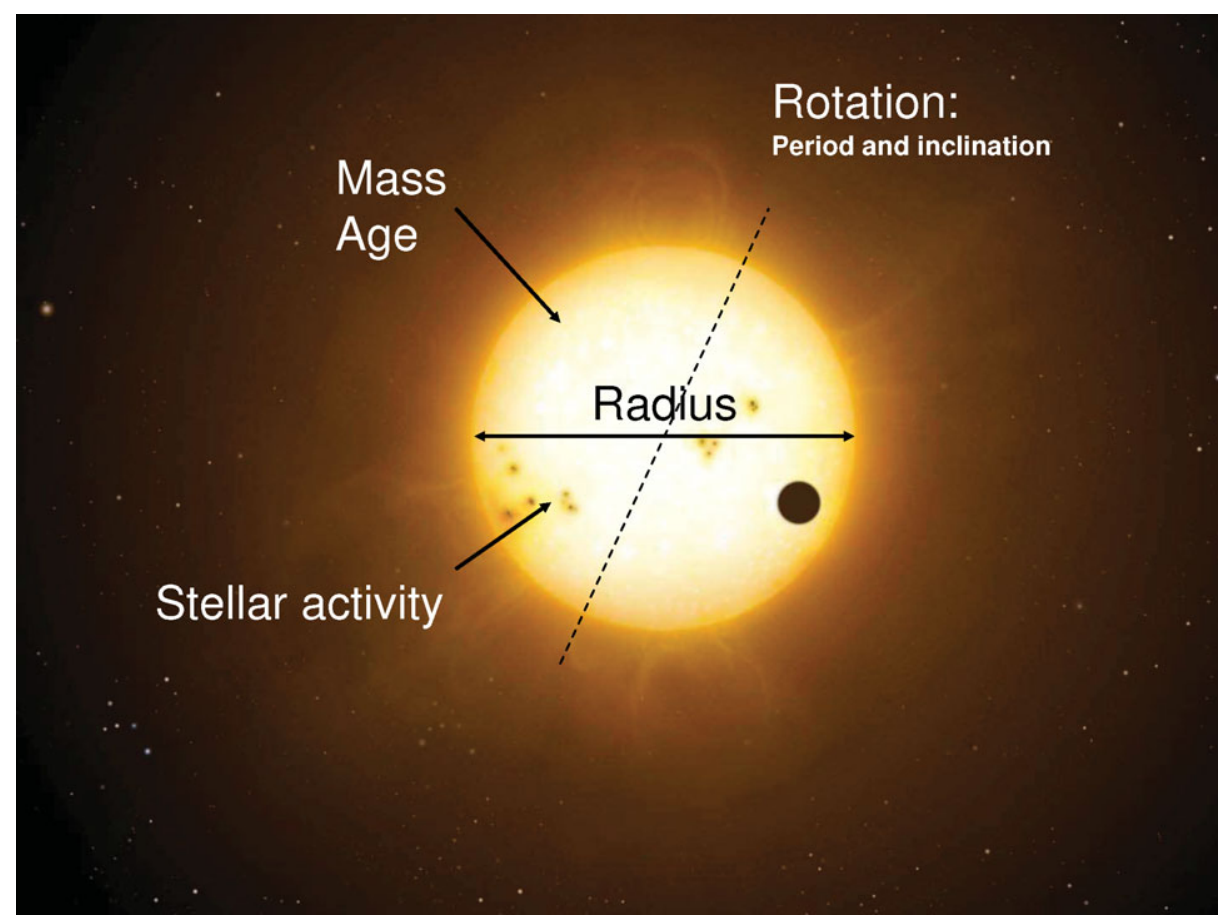

Figure 7. Using asteroseismology we are able to measure detailed basic properties of stars that have a transiting planet. Asteroseismology is expected to become a crucial tool for the Kepler mission when measuring stellar parameters.

$V=12.5$, and on evolved main-sequence stars (which have higher amplitudes) down to $V=13.5$. For subgiant stars we may reach even fainter. It should be noted that Kepler will be the first mission where asteroseismology will be applied to a large number of planet-hosting stars.

Finally, we note that accurate measurements of variations in the light travel times for planets orbiting classical pulsating stars (the pulsations are here used as accurate clocks) may provide a unique tool for the Kepler mission to detect a number of additional planets. The technique requires accurate measurements of phase variations of the pulsations (better than seconds) and the method will be most sensitive to long-period planets (1-2 years). For details on this technique we refer to Silvotti et al. (2007).

\section{References}

Aerts, C., Christensen-Dalsgaard, J., Cunha, M., \& Kurtz, D. W., 2008, Solar Phys., in the press [arXiv:0803.3527]

Arentoft, T., Kjeldsen, H., Bedding, T. R., et al. 2008, ApJ, in the press

Bedding, T. R. \& Kjeldsen, H. 2008, in: G. van Belle (ed.), 14th Cambridge Workshop on Cool Stars, Stellar Systems, and the Sun, ASP Conf. Ser., Vol. 384 (San Francisco: ASP), 21

Bedding, T. R., Kjeldsen, H., Butler, R. P., et al. 2004, ApJ 614, 380

Borucki, W. et al. 2008, these Proceedings

Christensen-Dalsgaard, J. 1993, in: T. M. Brown (ed.), Proc. GONG 1992: Seismic investigation of the Sun and stars, ASP Conf. Ser., Vol. 42 (San Francisco: ASP), 347

Christensen-Dalsgaard, J. 2004, Solar Phys. 220, 137 
Christensen-Dalsgaard, J., Arentoft, T., Brown, T. M., Gilliland, R. L., Kjeldsen, H., Borucki, W. J., \& Koch, D. 2007, Comm. in Asteroseismology 150, 350

Frandsen, S., Carrier, F., Aerts, C., et al. 2008, A\&A 394, L5

García, R. A., Turck-Chièze, S., Boumier, P., et al. 2005, A\&3A 442, 385

Gizon, L. \& Solanki, S. K. 2003, ApJ 589, 1009

Kjeldsen, H., Bedding, T. R., Butler, R. P., et al. 2005, ApJ 635, 1281

Silvotti, R., Schuh, S., Janulis, R., et al. Nature 449, 189

Ulrich, R. K., García, R. A., Robillot, J.-M. et al. 2000, A\&̋A 364, 799 